\title{
A Discussion on the Cultivation of Artistic Originality
}

\author{
Jianhong Yin \\ College of Art and Appareluages, Tianjin Polytechnical University, Tianjin 300160, China \\ E-mail: slimyin@163.com
}

\begin{abstract}
The artistic culture of Artists is reflected on the artistic works in the form of artistic emotion. Originality is the key in making a specific artistic works different from others. Innovative designs will be respected and admired by future generations. We are now confronted with the reality of the lack of originality and the inadequacy of creativity. In the process of basic technique learning, we should learn to think and focus on both learning and thinking, and learn to draw inferences about other cases from one instance so as to achieve high level in learning.
\end{abstract}

Keywords: Originality, Innovation, Artistic design, Sentiment

We are living in a new era of information explosion and competition, an ear that needs thinking reform, and a world that requires you to be different, outstanding, special and attractive.

Originality is a creative and extraordinary way of thinking. When we say that somebody is original in thinking, we mean that his or her way of thinking is different from ordinary people and he or she always breaks conventions. Originality is a representation of soul activities and has always been the root that distinguishes a person form others. The nature of art is to create. All creators hope that they could design works that are better than their former works and are distinctive. The artistic works have various characteristics and are irreplaceable. Even artistic works designed by one artist could not replace each other because each is special and irreplaceable. This kind of creation originates from originality. Creators need more originality (Edit group of Art Introduction, 2001). At present, most of the creators like to absorb nutrition and experience from traditional culture. However, they lack subjective sense of recognizing and are inadequate in the power of dominating matters. The inadequacy of originality causes the artistic works to be lacking features and to fail in generating distinctive personal characteristics, which is unfavorable in forming long-lasted creation life-force. When constructing a maim body, the base shall be solid. At the initial stage of cultivation of originality, "imitation" is a necessary road. However, ultimately, one has to find their won design style. The aim of the cultivation of creativity and the possession of novel originality is to cultivate artists rather than craftsmen.

\section{Which is more important, technique or thinking?}

Technique is a kind of capacity and is a capacity that one could learn from experience. Luo Dan believes that, "If without knowledge in volume, proportion, and color, and without ingenious hands, even the most enthusiastic feelings will be paralyzed." However, thinking is more important than knowledge and techniques. We all know that Still LifeVase with Fifteen Sunflowers and Self-portrait, the works of Vincent van Gogh, reflect impressionism. In his painting works at earlier stage, we could see his solid painting technique. However, his earlier works are not that special and distinctive. After going through the basic training, every one could do it. However, the works created by him at later stage were endowed with movement sense and flashing rhythm. From the grass and trees that he drew, we could fell the sense of life. The fierce friction not only comes from technique but also and more from the thinking of the author. Only those works that crash with thinking could live the test of time and then help to fix one's personal creation style so as to differ oneself from others and to make one's works special. Art comes from life but also exceeds the reality. The re-creation of the prototypes is a test for the artistic techniques of artists and is also and more a test for the imagination of artists. Hegel thinks, "As for ability, the most outstanding artistic ability is imagination." Artistic imagination is a kind of soul creation, is a spiritual freedom and is a kind of psychological ability of human beings that is with the least restriction. The significance of creative artistic imagination is determined by the nature of artistic creation themselves. If the excellent basic technique of Vincent van Gogh is the basis for his expression of personal disposition at the later stage, then the reason that he is respected by the future generations is out of his subjective analysis of matters, his splendid personality and his pursuit of personal style (Edit group of Art Introduction,2001).

Many people could not fully show their personal thoughts. The reason might be that they lack the capacity of independent thinking and the capacity to transform the thinking results into works. It is not difficult to solve the problems in techniques. Perseverance will prevail. However, it is hard to solve problems in thinking and awareness. It is a process for a person to learn how to think and this process is where a person accumulates knowledge and understands the knowledge. Firstly, a person shall be in possession of certain knowledge. The accumulation of knowledge shall be 
"focused" on certain aspect and at the same time, one should try to become a person with comprehensive knowledge. Knowledge from any field will conduce to opening the gate of thinking. Only after one possesses certain knowledge, one could learn to think, could be able to think, to make judgment on matters, and to form his or her own opinions, and ultimately represent his or her thoughts with artistic works. Technique is the base of creation and thinking the soul of creation. Soul is the genuine charm of artistic works. A good drawing is not that the drawing resembles the prototype, but resembles in spirit. A works that impresses people with its emotion will ever last.

\section{To feel the meaning of "it is better to teach how to fish than just to give fish" from "sentiment"}

Originality is a test for the comprehensive capacity of artists. Inspiration is indispensable in all arts and comes from inspiration source. This "source" refers to a tree, a grass, a flower, an important event, or a special memory; or it may come from beautiful music, a performance or a drama. Things that could impress soul would bring about a theme and then a series of works. Wang Fuzhi once said, "No matter it is poem or lengthy articles, they all focus on the meaning. Meaning is to works what general to an army. An army without a general is just a group of mobs." It is fortune that the readers and watchers recognize, value, and understand the theme of your works.

However, at the initial stage of searching for inspiration, redundant information is an interruption to the useful information. Therefore, it is significant to learn how to select useful information and discard those that are not. To solve this problem, one must start with the aspects one is most familiar with and tries to find novelty. Nevertheless, the pursuit of novelty shall not be just for realization of novelty. A works which is designed without inspiration and soul can never move others. A good works shall first move yourself and shall be designed and created with full enthusiasm and exciting mood. A works can move others only when it moves yourself.

In the activities of artistic creation, sentiment is a key point. When examining and commenting on a drawing, Gu Ningyuan relies on the heaven sentiment, object sentiment and human being sentiment. Only when one is outstanding, can observe the world and life in quiet, and understand sentiment, one can draw. Then what is "sentiment"? "Sentiment" is inspirational nature and is a way of thinking (Edit group of Art Introduction, 2001). We must first cultivate our interests and make clear what we are enthusiastic about and what can excite our thoughts. That is to say, we have to search for a thinking and creation mode that is appropriate to ourselves. Do not take such search as a waste of time. If the way, method and direction is right, what you will solve is not just a problem but you will find the way to solve all problems. If you find the right direction and understand sentiment, you will have the inspirational nature. Xu Zhimo did not read through books in America, but he did in Cambridge. Xu Zhimo believed that Cambridge opened his mind. Why would he have such feeling? The reason is that the seemingly easygoing life in Cambridge taught him to think. It is the same to us. "Your speech teaches me more than my 10-year's learning". Either we are having a class or having a chat, we must think about and discuss certain issue. In the process of thinking and discussion, when confronted with what we do not understand, we must go to search for materials. If we could be active in the process, what we solve will not only be the certain problems but our thoughts. It is important to attach the same significance to learning and thinking. A good teacher or a helpful friend who will guide you will be the key that helps to open the door to "thinking". A good teacher can teach you how to fish, enlighten your thoughts, open your mind, and help you to learn to draw inferences about other cases from one instance, and you will benefit deeply from such a teacher. A good teacher is just one aspect. It is you who are the protagonist. First of all, you must have profound interest in certain objects. To find the objects that you are interested in is the key for you to think. If you are obsessed with certain objects, you will unconsciously be absorbed in them and then will think and reason on the objects and problems. Works created by thinking and devotion will arouse others' common recognizing and win others' appreciation.

\section{To break the existing mode and search for new ideas}

When designing artistic works, one should pursue "new ideas" and break the existing mode. To obtain new ideas, one has to break the routine way of thinking. With the accumulation of knowledge and techniques, people will consciously or unconsciously form a kind of fixed thinking pattern. The fixed thinking pattern could help you to cultivate a certain type of design style. However, this fixed thinking pattern at the same time endangers you and blocks you from proceeding ahead. By the fixed thinking pattern, we will always think in certain mode, our thoughts develop towards one single direction, and we could not be teemed with imagination like a child. Naturally, blocked by this fixed thinking pattern, one could not design creative works. Therefore, we should frequently break the routine and existing thinking pattern, should observe things from different angles and perspectives and should think in a different way. We should think in the way as a child does. At least, we should try to think as a child does. It is a must that we dare to dream and to imagine.

A saying of Arabs is that "Personality determines everything". Therefore, the creation capacity of a human being is closely connected with the personality of the person. The following personality characteristics are conducive to helping a person to jump out of the routines and to accept new ideas.

Curious: be extremely curious about and eager to learn about the future things; dare to raise questions and dissension; 
be fond of independent thinking; be not willing to accept others' theories without thinking.

Tolerant: be tolerant about things and others; be able to endure and to accept oblique and contradictive things and ideas.

Optimistic: be able to treat things in positive and active mood; and assume the attitude of no failure but just feedback;

Positive: have strong inner movement motivation; be full of energy; have a wide interests; like challenges; tend to think independently; be not afraid of authority; be able to take initiative to question and stick to it wit persistence until the answers is found.

Absorbed: pursue uncommon aims; be absorbed in such pursuit, be indulged in it; forget food and eat and spend all his or her time in it.

Humorous: humor is a path for language thoughts; laugh is a good prescription to solve problems; it is easier for people who have the sense of humor, are fond of laughing and are happy to have inspiration and novel ideas than those who are passive.

Imaging: it is the marks of creative persons to have rich imagination power, trust intuition and like contemplation.

Of course, one who possesses the afore mentioned personality characteristics might not always be creative. However, it is sure that they live an elastic life, could master more psychological and environmental resources and could have more choices. With more choices, the chances of being successful will be more.

The art culture of artists is reflected on the artistic works in the form of artistic emotion. Originality is the key in making a specific artistic works different from others. Innovative designs will be respected and admired by future generations. In the process of basic technique learning, we should learn to reason and focus on both learning and thinking, and learn to draw inferences about other cases from one instance so as to achieve high level in learning.

\section{References}

Edit group of Art Introduction. (2001). Art Introduction. Beijing: Culture and Art Press.

Guo, Wenyu. (1998). Western Art History Compendium. Tianjin: Tianjin People's Art Press.

Lin, Yutang. The Vision of People with Wisdom: China and the World-Lin Yutang Collectanea (the second volume). International Culture Press Company 